\title{
Optimization of Linkage between North Carolina EMS and ED Data: EMS Naloxone Cases
}

\author{
Jonathan Fix ${ }^{1}$, Dennis Falls ${ }^{2}$, Scott Proescholdbell ${ }^{3}$, Amy Ising ${ }^{2}$, Tony Fernandez ${ }^{4}, 2$, Anna E. \\ Waller $^{2}$ \\ ${ }^{1}$ Epidemiology, UNC - Chapel Hill, Carrboro, North Carolina, United States, ${ }^{2}$ Emergency Medicine, UNC-CH, Chapel Hill, North Carolina, United \\ States, ${ }^{3}$ North Carolina Division of Public Health, Raleigh, North Carolina, United States, ${ }^{4}$ EMS Performance Improvement Center, Chapel Hill, North \\ Carolina, United States

\section{Objective}

To improve linkage between North Carolina's Emergency Medical Services (EMS) and Emergency Department (ED) data using an iterative, deterministic approach.

\section{Introduction}

The opioid overdose crisis has rapidly expanded in North Carolina (NC), paralleling the epidemic across the United States. The number of opioid overdose deaths in NC has increased by nearly 40\% each year since 2015 [1]. Critical to preventing overdose deaths is increasing access to the life-saving drug naloxone, which can reverse overdose symptoms and progression. Over 700 EMS agencies across NC respond to over 1,000,000 calls each year; naloxone administration was documented in over 15,000 calls in $2017[2]$.

Linking EMS encounters with naloxone administration to the corresponding ED visit assists in understanding the health outcomes of these patients. However, less than $66 \%$ of NC EMS records with naloxone administration in 2017 were successfully linked to an ED visit record. This study explored methods to improve EMS and ED data linkage, using a multistage process to maximize the number of correctly linked records while avoiding false linkages.

\section{Methods}

EMS data were provided by the EMS Performance Improvement Center [2] (EMSPIC); ED data were provided by NC DETECT [3]. Optimization of current EMS/ED linkage methods began by extracting a non-random subset of EMS encounters with naloxone administration between January 1, 2017 and November 30, 2017 from 12 NC counties, representing eastern, central and western regions and the overall linkage performance of the larger dataset. Records were eligible for linkage if EMS recorded that the patient was "treated and transported" to the ED. All records in the subset were manually reviewed in NC DETECT to identify corresponding ED visit records. This produced a "gold standard" dataset of linked EMS/ED records.

To evaluate linkage performance, we first identified all records eligible for linkage. Any EMS transport to either a hospital outside of NC or an NC ED not included in NC DETECT (e.g., military, VA and tribal hospitals) was excluded. Since existing linkage is performed daily and both EMS and ED records are updated over time to correct errors and missing data, existing linkage method s were re-run on updated data to evaluate the improvement provided solely by linking the most up- to-date data. Unlinked EMS records for which the encounter was an inter-facility transfer, transfer to helicopter transport, or the patient died during transfer were deemed ineligible for linkage, as these patients likely either bypassed or never made it to the ED.

To initially improve linkage quality, we updated the mapping file of EMS/ED destinations. An exact destination match was required for linkage and the EMS destination variable is recorded as free-text; thus, all variations of a destination name and spelling were identified and mapped to a standardized name. The maximum time difference between EMS drop-off and ED intake was then allowed to exceed 60 minutes, in iterations of 90, 120, 240, and 360 minutes. With each iteration, we compared the linked IDs with the gold standard dataset to identify false links.

Finally, a multistage linkage process was applied. First, deterministic linkage was run requiring exact matches for date of birth (DOB), sex/gender, and destination, and up to 360-minute difference between EMS/ED times. The unlinked records were then 
processed a second time, requiring exact matches for sex/gender and destination, DOB to be within $+/-10$ days or $+/-1$ year, and up to 60-minute difference between EMS/ED times.

This multistage process was then run for all 2017 EMS encounters with naloxone administration to ensure that the new method was not over fit to the data subset. Potential bias in the linkage was assessed by comparing the distributions of age (mean and median) and gender (\% male) among the linked and unlinked records in each dataset.vStatistical analyses were completed using SAS 9.4 (Cary, NC). Linkage was executed using SQL Server.

\section{Results}

Between 1/1/2017 and 11/30/2017, there were 14,793 EMS encounters with documented naloxone administration. Of these, 12,089 $(81.7 \%)$ were recorded as "treated and transported"; 1,906 EMS encounters were included in the 12-county subset. The average age of patients was 45.1 years among all naloxone encounters and 45.2 years in the subset. $57.5 \%$ of all encounters were male; $58.1 \%$ were male in the subset.

After removing EMS transports to non-NC or non-NC DETECT hospitals, the existing subset linkage was $61.8 \%(1,154 / 1,866)$. This included $38(2.0 \%)$ false positives, apparently caused by ED records purged since this linkage was conducted. When the existing methods were run against the most current data, linkage improved to $72.2 \%(1,389 / 1,866)$, reflecting an absolute improvement of $10.4 \%$ by simply using updated data. Only $1(0.05 \%)$ false positive was identified in this process.

Following removal of unlinked inter-facility transfers, deaths during EMS transport, and transfers to helicopters, the records eligible for linkage dropped to 1,781. Linkage improved to $79.5 \%(1,417 / 1,781)$ when hospital names were standardized. Linkage using standardized hospital names and relaxing the EMS/ED time difference performed at the following levels: $82.3 \%$ at 90 minutes, $83.3 \%$ at 120 minutes, $87.9 \%$ at 240 minutes, and $89.4 \%$ at 360 minutes. Even when using the most relaxed time difference (+/360 minutes), only one false positive was identified, the same produced during initial linkage at $+/-60$ minutes. The final multistage method produced linkage of $91.0 \%(1,620 / 1,781)$, with no additional false positives.

Applying the initial methods to the statewide EMS dataset produced linkage of $64.8 \%$. The multistage linkage process performed nearly identically on statewide data as observed for the subset, at $91.1 \%$. For statewide data, the age of linked patients was younger $($ mean $=44.7$ years $[S D=18.4]$, median $=41.0$ years $)$ than that of unlinked patients $($ mean $=48.0$ years $[\mathrm{SD}=19.3]$, median $=$ 47.0 years). Additionally, linked patients were more likely to be male (58.1\%) when compared to unlinked patients $(54.2 \%)$.

\section{Conclusions}

High quality linkage between EMS and ED records is essential for research and public health surveillance examining health outcomes. Using a multistage process, we improved the linkage of EMS encounters with documented naloxone administration to ED visits in North Carolina in 2017 from $64.8 \%$ to 91.1\%, with less than $0.05 \%$ false positive rate. This improved linkage will facilitate future analyses of relationships between exposures during EMS encounters and outcomes experienced in hospitals. Future research should evaluate the generalizability of this linkage methodology to all EMS records, not just those with naloxone administration, as well as to pre-2017 data. Implementation of probabilistic linkage or machine learning as a final stage in a multistage process may further improve linkage outcomes, overcoming missing data or unpredictable errors in the data.

\section{Acknowledgement}

Funding provided by the CDC National Center for Injury Prevention and Control Enhanced State Opioid Overdose Surveillance (ESOOS) grant to the NC Division of Public Health (grant 5NU17CE924902).

\section{References}

1. Kansagra SM, Cohen MK. 2018. The Opioid Epidemic in NC: Progress, Challenges, and Opportunities. $N C$ Med J. 79(3), 157-62. PubMed https://doi.org/10.18043/ncm.79.3.157

2. EMS Performance Improvement Center. About EMSPIC. https://www.emspic.org/about.

3. NC DETECT. Background. http://ncdetect.org/background/ 